\title{
absorção, Translocação e Metabolismo do Glyphosate por Plantas Tolerantes e Suscetiveis a este Herbicida ${ }^{1}$
}

\author{
Absorption, Translocation and Metabolism of Glyphosate by Plants Tolerant and Susceptible to \\ this Herbicide
}

\author{
MONQUERO, P.A. ${ }^{2}$, CHRISTOFFOLETI, P.J. ${ }^{3}$, OSUNA, M.D. ${ }^{4}$ e DE PRADO, R.A. ${ }^{4}$
}

\begin{abstract}
RESUMO - O objetivo deste trabalho foi caracterizar a absorção foliar, a translocação e o metabolismo do ${ }^{14} \mathrm{C}$-glyphosate pelas plantas daninhas Commelina benghalensis, Ipomoea grandifolia e Amaranthus hybridus. O glyphosate foi aplicado através de quatro gotas de $0,5 \mu \mathrm{L}$ de uma solução contendo o produto comercial, na dose de campo de 720 g e.a. ha ${ }^{-1}$ em mistura com ${ }^{14} \mathrm{C}$-glyphosate, na face adaxial da segunda folha verdadeira das plantas estudadas. As avaliações foram feitas a 2, 4, 8, 12, 24, 48 e 72 horas após o tratamento com o herbicida (HAT) para os estudos de absorção e translocação e 72 HAT para os estudos de metabolismo. Os resultados de absorção e translocação demonstraram que mais de $90 \%$ do glyphosate aplicado foi absorvido por A. hybridus até 72 HAT. A maior parte do herbicida permaneceu na folha tratada, com uma taxa de translocação em torno de $25 \%$ do glyphosate aplicado. Em I. grandifolia, 80\% do herbicida foi absorvido até 72 HAT, porém houve menor translocação - somente 2,$2 ; 3,5 ;$ e $4,6 \%$ de ${ }^{14} \mathrm{C}$ glyphosate absorvido estava presente na parte aérea, no caule e na raiz. C. benghalensis apresentou uma taxa de absorção de 66\% até 72 HAT, sendo, portanto, a penetração diferencial do herbicida glyphosate um provável mecanismo de tolerância desta planta daninha. Nessa avaliação, 39\% do glyphosate estava presente na folha tratada e 15,2 e 11,6\% na parte aérea e raiz, respectivamente. Nos estudos de metabolismo foi detectado o metabólito ácido aminometilfosfônico (AMPA) apenas em $C$. benghalensis, sendo, portanto, o metabolismo diferencial um possivel mecanismo de tolerância desta planta daninha. Os resultados obtidos nesta pesquisa permitem concluir que os mecanismos de tolerância de C. benghalensis ao glyphosate são a absorção diferencial e o metabolismo do herbicida pela planta daninha. Em I. grandifolia a tolerância ocorre devido a uma menor translocação do herbicida, não havendo evidências de metabolismo diferencial do herbicida por esta planta daninha.
\end{abstract}

Palavras-chave: tolerância, Commelina benghalensis, Ipomoea grandifolia, Amaranthus hybridus, AMPA.

\begin{abstract}
The objective of this research was to characterize foliar absorption, translocation and metabolism of ${ }^{14} \mathrm{C}$-glyphosate by the weeds Commelina benghalensis, Ipomoea grandifolia, and Amaranthus hybridus. Glyphosate was applied through four drops of $0.5 \mu \mathrm{L}$ of a solution containing the commercial product, at the recommended dose of $720 \mathrm{~g}$ a.e.ha $\mathrm{h}^{-1}$ mixed with ${ }^{14} \mathrm{C}$-glyphosate, on the adaxial surface of the second true leaf of the studied plants. The evaluations were conducted at 2, 4, 8, 12, 24, 48 and 72 hours after herbicide treatment (HAT) for the absorption and translocation experiments and 72 HAT for the metabolism experiment. The absorption and translocation results showed that $\boldsymbol{A}$. hybridus absorbed more than $90 \%$ of the glyphosate applied after 72 HAT. The major part of the herbicide remained in the treated leaf, with a glyphosate translocation rate of 25\%. In I. grandifolia, $80 \%$ of the herbicide was absorbed after 72 HAT; however, there was a lower translocation, and only 2.2; 3.5 and $4.6 \%$ of the ${ }^{14} \mathrm{C}-$ glyphosate were present in the shoot, branch and root, respectively. C. benghalensis presented an absorption rate of 66\% after 72 HAT, thus differential glyphosate penetration was the mechanism responsible
\end{abstract}

Recebido para publicação em 3.11.2003 e na forma revisada em 10.9.2004.

2 Professora substituta do Departamento de Recursos Naturais do Centro de Ciências Agrárias - CCA-UFSCar, Rodovia Anhanguera, 13600-000 Araras-SP, <pamonque @ hotmail.com>. ${ }^{3}$ Professor Associado do Departamento de Produção Vegetal da Universidade de São Paulo, Escola Superior de Agricultura “Luiz de Queiroz”, 13419-900 Piracicaba-SP, Brasil. ${ }^{4}$ Departamento de Química y Edafologia, Universidad de Córdoba-Espanha. 
for tolerance of this weed. In this evaluation, 39\% of the glyphosate was present in the treated leaf and 15.2 and $11.6 \%$ in the shoot and root, respectively. In the metabolism studies, the metabolite aminomethylphosphonic acid (AMPA) was detected only in $\mathbf{C}$. benghalensis, with differential metabolism being the mechanism of tolerance of this weed. The overall conclusion of this research is that glyphosate tolerance of $\mathbf{C}$. benghalensis is due to the differential absorption and translocation of the herbicide by the weed. In I. grandifolia, the tolerance is due to the lower translocation of the herbicide, with no data evidence that differential metabolism of the herbicide occurs in this weed.

Key words: $\quad$ tolerance, Commelina benghalensis, Ipomoea grandifolia, Amaranthus hybridus, AMPA.

\section{INTRODUÇÃO}

O glyphosate é um herbicida aplicado em pós-emergência, cuja eficácia é dependente de processos como a retenção da molécula na superfície foliar, a penetração foliar, a translocação na planta até o sítio de ação e a inibição da enzima-alvo, a enolpiruvilshiquimatoP-sintetase (EPSPs) (Kirkwood \& Mckay, 1994).

A absorção do glyphosate é um processo bifásico que envolve uma rápida penetração inicial através da cutícula, seguida por uma absorção simplástica lenta. A duração desse processo é dependente de vários fatores, como espécie e idade da planta, condições ambientais e concentração do herbicida na calda e do surfatante. Uma vez que o glyphosate penetra na planta através da cutícula e membrana plasmática dos tecidos fotossintetizantes, é necessário que ocorra a translocação simplástica, através de tecidos vasculares, para os sítios-alvo do herbicida (Satichivi et al., 2000). O movimento do glyphosate pelo floema segue a mesma rota dos produtos da fotossíntese (açúcares), ocorrendo das folhas fotossinteticamente ativas em direção às partes das plantas que utilizam esses açúcares para crescimento, manutenção e metabolismo, ou armazenamento para uso futuro, como, por exemplo, raízes, tubérculos, rizomas, folhas jovens e zonas meristemáticas. A quantidade de açúcar translocada para cada uma dessas partes muda durante o ciclo de vida da planta e, conseqüentemente, influencia o movimento do herbicida. Portanto, condições que favoreçam a fotossintese auxiliam também a translocação do glyphosate (Dellacioppa et al., 1986). A translocação representa um processo essencial para a eficácia do herbicida (Wanamarta \& Penner, 1989). Da mesma forma, a absorção e o metabolismo também podem afetar a suscetibilidade de uma planta ao glyphosate.

De modo geral, o metabolismo dos herbicidas nas plantas ocorre em quatro fases (Colen, 1994). A fase I (transformação) é caracterizada pela alteração na estrutura química do herbicida, causada por reações de oxidação, redução e hidrólise. Na fase II (conjugação) muitos herbicidas são rapidamente conjugados ao açúcar por ponte glicosídica. Esse processo é catalisado pela glicosiltransferase. Os herbicidas podem ser também conjugados a glutationa pela glutationa-Stransferase, resultando, assim, em conjugados menos fitotóxicos e mais solúveis em água. Na fase III (compartimentalização) ocorre o transporte do herbicida para dentro do vacúolo ou matriz extracelular, e na fase IV há o processamento completo desses compostos. Nos solos e nas plantas, o principal metabólito do glyphosate é o ácido aminometilfosfônico (AMPA), detectado em algumas plantas naturalmente tolerantes a este herbicida, como Agropyron repens e Equisetum arvense (Coupland, 1985; Dyer, 1994).

A maioria dos casos observados de tolerância de plantas ao glyphosate não teve seus mecanismos elucidados. O entendimento desses mecanismos é fundamental para que alternativas de manejo sejam planejadas ou para melhorar a eficácia do produto. O objetivo deste experimento foi elucidar os principais mecanismos de tolerância das plantas C. benghalensis e I. grandifolia ao herbicida glyphosate, por meio da análise de absorção, translocação e metabolismo diferencial do herbicida nestas espécies. 


\section{MATERIAL E MÉTODOS}

Os estudos dos mecanismos de tolerância de plantas ao glyphosate foram conduzidos nos laboratórios e instalações do Departamento de Química Agrícola e Edafologia da Universidad de Córdoba, campus de Rabanales, Espanha, sob a orientação do Prof. Dr. Rafael de Prado Amián.

\section{Preparo da solução de glyphosate radiomarcado}

Glyphosate marcado com carbono $14\left({ }^{14} \mathrm{C}\right)$ no radical phosphonomethylene (C3), apresentando atividade específica de $200 \mathrm{GBq}$ $\mathrm{mmol}^{-1}$ e 98\% de pureza, foi empregado nos experimentos de absorção, translocação e metabolismo. Nos estudos de absorção e translocação foi preparada uma solução com volume de $100 \mu \mathrm{L}$, contendo 163,5 KBq; e para os estudos de metabolismo foi preparada uma solução de $250 \mathrm{KBq}$. Estas soluções foram preparadas diluindo-se o glyphosate marcado em uma calda contendo o glyphosate comercial Roundup ${ }^{\circledR}$, de tal forma que a concentração final do herbicida seria equivalente à dose comercial de 720 g e.a. ha ${ }^{-1}$, com um volume de calda de 300 L.ha $^{-1}$. Um total de $3,33 \mathrm{KBq}$ (estudos de absorção e translocação) e 5,0 KBq (metabolismo) foi aplicado em cada planta.

\section{Metodologia para os estudos de absorção e translocação do glyphosate}

Sementes de C. benghalensis, I. grandifolia e A. hybridus foram semeadas separadamente em vasos com capacidade de $200 \mathrm{~mL}$, contendo areia e matéria orgânica na proporção de 3:1. Os vasos foram mantidos em uma câmara de crescimento com temperatura dia/noite de $32 / 18{ }^{\circ} \mathrm{C}$, respectivamente, fotoperíodo de 16 horas de luz ( $350 \mu \mathrm{mol} \mathrm{m} \mathrm{m}^{-2} \mathrm{~s}^{-1}$ ) e umidade relativa constante de $80 \%$, irrigando-se diariamente, visando a manutenção da umidade satisfatória do solo. Estabeleceu-se a densidade de duas plantas por vaso, buscando uniformizar a área foliar e o estádio de desenvolvimento, a fim de minimizar a variabilidade experimental. As plantas foram tratadas em laboratório, quando apresentavam quatro folhas verdadeiras, com ${ }^{14} \mathrm{C}$-glyphosate em mistura com glyphosate comercial, aplicado sobre a face adaxial da segunda folha, na forma de quatro gotas de $0,5 \mu \mathrm{L}$ cada uma, usando um microaplicador (Hamilton PB6000 Dispenser, Hamilton Co., Reno NV), totalizando 3,33 KBq em cada planta.

Após a aplicação do herbicida, a absorção e translocação foram estudadas em diferentes períodos $(2,4,8,12,24,48$ e 72 horas após tratamento com o herbicida (HAT)), nos quais as plantas permaneceram em câmara de crescimento, nas condições supracitadas. Após cada período, as plantas foram retiradas dos vasos e fracionadas em diferentes partes (folha tratada, demais folhas, caule e raiz), com o objetivo de quantificar a radioatividade em cada uma delas. As folhas tratadas foram lavadas com $3 \mathrm{~mL}$ de metanol 80\%, a fim de eliminar o herbicida não absorvido. O líquido proveniente dessa lavagem foi misturado com o líquido de cintilação, sendo levados para posterior medição da radioatividade residual. As outras partes das plantas foram secas separadamente por 48 horas a $50{ }^{\circ} \mathrm{C}$ e submetidas posteriormente à combustão em um oxidador Packard Sample Oxidizer, modelo 307.

A combustão se realizou em uma atmosfera rica em oxigênio, de forma que todo ${ }^{14} \mathrm{C}$ presente nas amostras fosse convertido na forma de ${ }^{14} \mathrm{CO}_{2}$. ${ }^{14} \mathrm{CO}_{2}$ desprendido passou através de uma coluna que continha Carbosob como suporte, substância capaz de retê-lo e transformá-lo em carbamato. As amostras de carbamato resultantes de cada tratamento foram coletadas individualmente e adicionadas a $5 \mathrm{~mL}$ de líquido de cintilação (Permafluor). A radioatividade contida nesse meio foi posteriormente medida em espectrômetro de cintilação líquida, modelo Beckman LS 6000 TA.

A quantidade de herbicida absorvida foi calculada pela equação:

$$
\% \quad H \text { abs }=\frac{{ }^{14} \text { C tec.ox. }}{{ }^{14} \text { C tec.ox. }+{ }^{14} \text { C lav. }} \times 100
$$

em que $H$ abs = herbicida absorvido pelas plantas; ${ }^{14} \mathrm{C}$ tec.ox. = quantidade $\mathrm{de}{ }^{14} \mathrm{C}$ detectada nos tecidos oxidados; e ${ }^{14} \mathrm{C}$ lav. = quantidade de ${ }^{14} \mathrm{C}$ detectada na lavagem da folha tratada.

Planta Daninha, Viçosa-MG, v. 22, n. 3, p. 445-451, 2004 
O experimento foi conduzido em delineamento inteiramente casualizado, com quatro repetições para cada período de avaliação. O experimento foi repetido duas vezes. A radioatividade presente em todas as partes das plantas, exceto na folha tratada, foi considerada como translocada. Foram calculadas as médias das quatro repetições, juntamente com os respectivos desvios-padrões.

\section{Metodologia para os estudos do metabolismo de glyphosate}

Plantas de C. benghalensis, I. grandifolia e A. hybridus se desenvolveram em câmara de crescimento, nas mesmas condições descritas nos estudos de absorção e translocação. A mistura de glyphosate comercial e ${ }^{14} \mathrm{C}$-glyphosate foi aplicada sobre a superfície adaxial da segunda folha definitiva de cada planta, na forma de quatro gotas de $0,5 \mu \mathrm{L}$ cada uma, usando um microaplicador (Hamilton PB6000 Dispenser, Hamilton Co., Reno NV), totalizando $5,5 \mathrm{KBq}$ por planta.

Seguindo a aplicação de ${ }^{14} \mathrm{C}$-glyphosate, as plantas foram transferidas novamente para câmara de crescimento e avaliadas a 72 HAT. As folhas tratadas foram lavadas com metanol $80 \%$, para medir a radioatividade não penetrada na folha. A seguir as plantas foram separadas em parte aérea e raiz; esta última não foi utilizada neste experimento, já que no período máximo de avaliação havia ainda pouca radioatividade presente no sistema radicular. As partes aéreas foram inicialmente maceradas na presença de $\mathrm{N}_{2}$ líquido e homogeneizadas usando $3 \mathrm{~mL}$ de metanol $80 \%$. A seguir, foram submetidas à centrifugação ( $14.000 \mathrm{~g}, 4^{\circ} \mathrm{C}$ por 10 minutos) e alíquotas do sobrenadante foram analisadas, calculando-se a radioatividade. Após separar o precipitado do sobrenadante, o primeiro foi novamente lavado com metanol $80 \%$ até que não fosse mais possivel extrair radioatividade (repetiu-se este processo pelo menos três vezes). Os sobrenadantes de cada uma das amostras foram secos por uma corrente de $\mathrm{N}_{2}$, a uma pressão de $10 \mathrm{Kpa}$ e temperatura de $40^{\circ} \mathrm{C}$. O extrato seco foi ressuspendido em $500 \mu \mathrm{L}$ de metanol 80\%, e cerca de $250 \mu \mathrm{L}$ foram analisados por cromatografia de camada delgada, baseando-se no trabalho de Sprankle et al. (1978). Foram utilizadas placas de sílica-gel de 20 × $20 \mathrm{~cm}$ e
250 um (Merck, Sílica gel 60); como fase móvel utilizou-se uma mistura de etanol: água: $15 \mathrm{~N}$ $\mathrm{NH}_{4} \mathrm{OH}$ : TCA: $17 \mathrm{~N}$ ácido acético. As zonas radioativas, com os respectivos valores de Razão de Fluxo (Rf), foram detectadas por um radioanalisador Berthol, LB 2821, com suporte informático do programa Berthol Chroma-1D, instalado em um computador conectado ao analisador. Os picos obtidos pela cromatografia foram separados e identificados de acordo com os valores de $(\mathrm{Rf})$ disponíveis (glyphosate $=0,24$ e ácido aminometilfosfônico AMPA=0,45), segundo Sprankle et al. (1978).

\section{RESULTADOS E DISCUSSÃO}

\section{Absorção e translocação do glyphosate}

Em A. hybridus a absorção aumentou com o decorrer do tempo após aplicação; a 72 HAT pôde se observar a penetração de $93,5 \%$ de glyphosate (Figura 1). A translocação aumentou de maneira efetiva a partir de 12 HAT até 72 HAT, quando cerca de $69,3 \%$ do ${ }^{14} \mathrm{C}$-glyphosate estava presente na folha tratada (não translocado) e aproximadamente 7,2 ; 11,2 ; e $6,7 \%$ de glyphosate encontravam-se na parte aérea, no caule e na raiz, respectivamente (Tabela 1 ).

A absorção de ${ }^{14} \mathrm{C}$-glyphosate em Sorghum halepenses e Elytrigia repens variou de 7 a $74 \%$ durante os períodos de 48 e 120 horas de exposição (Devine et al., 1983; Mcwhorter et al., 1980; Wills, 1978).

Em I. grandifolia, a penetração de glyphosate foi de $80 \%$ (Figura 1), porém a taxa de translocação foi a menor quando comparada com a de todas as plantas estudadas; a 72 HAT, $68,5 \%$ do herbicida absorvido estava presente na folha tratada e 2,$2 ; 4,3$; e $4,9 \%$ foram observados na parte aérea, no caule e na raiz, respectivamente (Tabela 2). Chachalis et al. (1999) observaram o controle ineficaz de Ipomoea spp. pelo herbicida glyphosate, sugerindo que, em culturas transgênicas de soja e algodão resistentes ao glyphosate, o controle de Ipomoea spp. deve ser feito com herbicidas alternativos.

Em condições favoráveis, o glyphosate penetra rapidamente nas folhas. Sprankle et al. (1975) aplicaram ${ }^{14} \mathrm{C}$-glyphosate na concentração de $4,4 \mathrm{~g} \mathrm{~L}^{-1}$, contendo $0,8 \%$ de 
surfatante, na planta Agropyron repens. Em quatro horas, $34 \%$ do glyphosate aplicado havia penetrado, mas durante as próximas 44 horas o processo foi muito lento. Trabalhando com folhas de Apocynum cannabium, Schultz \& Burnside (1980) encontraram taxas de absorção de 19, 36 e 53\% nos períodos de 12 horas, 3 e 12 dias, respectivamente. Os valores de translocação apresentados a seguir foram retirados do trabalho de Sandberg et al. (1980) e correspondem ao ${ }^{14} \mathrm{C}$ translocado para fora da folha tratada com ${ }^{14} \mathrm{C}$-glyphosate após 14 dias do tratamento: Cirsium arvensis $(3,5 \%)$, C. sepium $(21,6 \%)$, I. purpurea $(6,5 \%) \mathrm{e}$ $P$. convolvulus $(5,0 \%)$ - estes valores foram expressos em porcentagem do ${ }^{14} \mathrm{C}$ aplicado.

A planta daninha $C$. benghalensis apresentou absorção inicial relativamente lenta, com aumento a partir das 24 HAT; a 72 HAT a taxa de absorção foi de $66,1 \%$ do ${ }^{14} \mathrm{C}$-glyphosate aplicado (Figura 1). Portanto, um dos mecanismos de tolerância desta planta daninha é a absorção diferencial do herbicida, que foi a menor obtida entre as plantas estudadas. Entretanto, parece não haver barreiras para a translocação, já que a 72 HAT foram encontrados $15,1 \%$ do glyphosate na parte aérea e $11,2 \%$ nas raízes (Tabela 3 ).

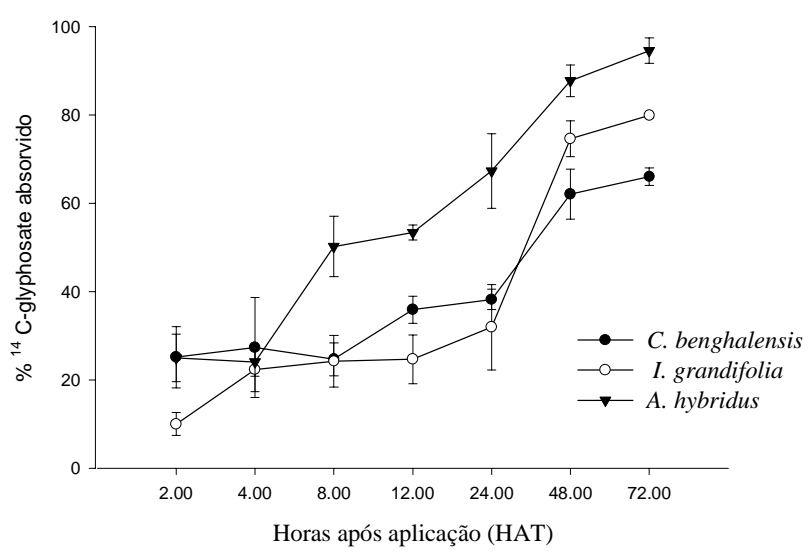

Figura 1 - Absorção de glyphosate nas plantas de C. benghalensis, I. grandifolia e A. hybridus.

Tabela 1 - Quantidade do ${ }^{14} \mathrm{C}$ glyphosate translocada (\% do ${ }^{14} \mathrm{C}$ aplicado) por Amaranthus hybridus, em função do tempo após a aplicação (HAT). Os valores de desvio-padrão estão entre parênteses

\begin{tabular}{|c|c|c|c|c|}
\hline HAT & Folha tratada & Folha & Caule & Raiz \\
\hline 2 & $24,55( \pm 2,80)$ & $0,31( \pm 0,11)$ & $0,26( \pm 0,18)$ & $0,16( \pm 0,05)$ \\
\hline 4 & $23,62( \pm 0,08)$ & $0,23( \pm 0,15)$ & $0,08( \pm 0,04)$ & $0,21( \pm 0,08)$ \\
\hline 8 & $48,31( \pm 1,40)$ & $0,62( \pm 0,36)$ & $0,90( \pm 0,42)$ & $0,93( \pm 0,31)$ \\
\hline 12 & $51,44( \pm 11,00)$ & $0,50( \pm 0,30)$ & $1,15( \pm 0,15)$ & $1,20( \pm 0,26)$ \\
\hline 48 & $58,57( \pm 1,40)$ & $2,73( \pm 1,12)$ & $3,06( \pm 1,59)$ & $2,97( \pm 1,52)$ \\
\hline 72 & $70,38( \pm 4,30)$ & $3,79( \pm 3,52)$ & $5,30( \pm 2,77)$ & $8,31( \pm 3,44)$ \\
\hline & $69,32( \pm 2,70)$ & $7,27( \pm 4,83)$ & $11,25( \pm 7,44)$ & $6,72( \pm 3,44)$ \\
\hline
\end{tabular}

Tabela 2 - Quantidade do ${ }^{14} \mathrm{C}$ glyphosate translocada (\% do ${ }^{14} \mathrm{C}$ aplicado) por Ipomoea grandifolia, em função do tempo após a aplicação (HAT). Os valores de desvio-padrão estão entre parênteses

\begin{tabular}{|c|c|c|c|c|}
\hline HAT & Folha tratada & Folha & Caule & Raiz \\
\hline 2 & $9,10( \pm 2,62)$ & $0,31( \pm 0,19)$ & $0,22( \pm 0,11)$ & $0,71( \pm 0,51)$ \\
\hline 4 & $21,53( \pm 2,10)$ & $0,29( \pm 0,14)$ & $0,16( \pm 0,03)$ & $0,32( \pm 0,01)$ \\
\hline 8 & $22,14( \pm 2,10)$ & $0,54( \pm 0,12)$ & $0,64( \pm 0,20)$ & $1,53( \pm 0,48)$ \\
\hline 12 & $22,21( \pm 9,30)$ & $0,55( \pm 0,14)$ & $0,66( \pm 0,07)$ & $1,54( \pm 0,14)$ \\
\hline 24 & $31,42( \pm 10,5)$ & $1,05( \pm 0,21)$ & $0,83( \pm 0,19)$ & $1,81( \pm 0,29)$ \\
\hline 48 & $69,90( \pm 1,06)$ & $2,66( \pm 0,34)$ & $1,85( \pm 0,73)$ & $2,09( \pm 0,39)$ \\
\hline 72 & $68,32( \pm 4,03)$ & $2,23( \pm 0,20)$ & $4,36( \pm 2,73)$ & $4,91( \pm 0,90)$ \\
\hline
\end{tabular}


Tabela 3 - Quantidade do ${ }^{14} \mathrm{C}$ glyphosate translocada (\% do ${ }^{14} \mathrm{C}$ aplicado) por Commelina benghalensis, em função do tempo após a aplicação (HAT). Os valores de desviopadrão estão entre parênteses

\begin{tabular}{|c|c|c|c|}
\hline HAT & Folha tratada & Folha & Raiz \\
\hline 2 & $24,08( \pm 2,62)$ & $0,40( \pm 0,20)$ & $0,34( \pm 0,02)$ \\
\hline 4 & $26,14( \pm 8,40)$ & $0,52( \pm 0,11)$ & $0,36( \pm 0,08)$ \\
\hline 8 & $23,29( \pm 2,70)$ & $0,54( \pm 0,21)$ & $0,22( \pm 0,02)$ \\
\hline 12 & $24,04( \pm 8,70)$ & $0,64( \pm 0,01)$ & $0,32( \pm 0,02)$ \\
\hline 24 & $35,36( \pm 10,23)$ & $1,41( \pm 0,71)$ & $0,74( \pm 0,16)$ \\
\hline 48 & $53,98( \pm 10,23)$ & $1,44( \pm 0,60)$ & $6,70( \pm 2,30)$ \\
\hline 72 & $68,32( \pm 4,03)$ & $15,10( \pm 1,70)$ & $11,23( \pm 0,68)$ \\
\hline
\end{tabular}

\section{Estudo do metabolismo do glyphosate}

Não foram detectados metabólitos do glyphosate (AMPA) nos extratos das plantas de I. grandifolia e A. hybridus, a 72 HAT do tratamento com ${ }^{14} \mathrm{C}$-glyphosate. Na Tabela 4 observa-se que 70,3 e 82,9\% da radioatividade extraída destas plantas daninhas foi encontrada na forma do herbicida glyphosate, apresentando pico-padrão com valores de Rf variando de 0,24 a 0,32 (dados não mostrados). Análises de extrato provenientes de plantas de $C$. benghalensis indicaram 40,8\% de metabolismo do herbicida (Tabela 4). Nesta planta daninha a análise a 72 HAT indicou dois picos diferentes, com valores de Rf de 0,24 e de 0,45. De acordo com Sprankle et al. (1978), estes valores correspondem aos valores de $\mathrm{Rf}$ do ${ }^{14} \mathrm{C}$ glyphosate-padrão e do ácido aminometilfosfônico (AMPA), respectivamente. A rota de degradação deste herbicida em plantas superiores não é bem compreendida. Alguns estudos têm sugerido que o metabolismo de glyphosate não ocorre (Mallik et al., 1989), enquanto outros mostram que a transformação química deste herbicida pode ocorrer.

Os resultados publicados sobre metabolismo de glyphosate são divididos em dois grupos: aqueles que não encontraram metabolismo e aqueles estudos nos quais foram detectados metabólitos (principalmente o AMPA). A inabilidade para detectar metabólitos em extratos de plantas não significa que não houve metabolismo. A decomposição do herbicida para $\mathrm{CO}_{2}$ pode envolver a subseqüente perda de glyphosate pela planta. Por outro lado, perda de glyphosate (ou de ${ }^{14} \mathrm{C}$ ) não
Tabela 4 - Identificação e quantificação, nos extratos das plantas, de glyphosate e de AMPA com relação à radioatividade aplicada a 72 HAT

\begin{tabular}{|l|c|c|}
\hline \multicolumn{1}{|c|}{ Planta } & Glyphosate & AMPA \\
\hline C. benghalensis & 30,9 & 40,8 \\
\hline I. grandifolia & 70,3 & nd \\
\hline A. hybridus & 82,9 & nd \\
\hline
\end{tabular}

Obs.: glyphosate $(\mathrm{Rf}=0,24-0,32)$, AMPA $(0,41-0,44)$, nd $=$ não detectado.

necessariamente significa uma perda degradativa. Este herbicida, como outros herbicidas móveis no floema, é conhecido por ser exsudado das raízes e folhas tratadas na forma de molécula intacta (Rodrigues et al., 1982).

Komoba et al. (1992) observaram metabolismo de glyphosate a AMPA em algumas plantas, como soja, trigo, milho e tabaco. Exsudatos de raízes de Agropyron repens examinados por Coupland \& Caseley (1979) oito dias após o tratamento com glyphosate radioativo através de cromatografia de camada delgada - apresentaram apenas uma área radioativa que correspondia ao herbicida. Resultado similar foi encontrado por Rodrigues et al. (1982) no estudo de metabolismo em trigo. Sandberg et al. (1980) observaram metabolismo deste herbicida em Ipomoea purpurea e Cirsium arvense, sendo o metabólito AMPA encontrado em uma taxa de $11 \%$.

Em algumas espécies de plantas o glyphosate é metabolizado, enquanto em outras ele é detectado como molécula intacta. Essas diferenças podem ocorrer por diversas razões, e pesquisas usando técnicas de análise e identificação mais apuradas são necessárias para se estabelecer se esses efeitos ocorrem realmente devido a um metabolismo diferencial do glyphosate.

Os resultados obtidos nesta pesquisa permitem concluir que os mecanismos de tolerância de $C$. benghalensis ao glyphosate são a absorção diferencial e o metabolismo diferencial do herbicida a AMPA. Em I. grandifolia, a tolerância ocorre devido a uma menor translocação do herbicida.

\section{AGRADECIMENTOS}

À FAPESP, pelo apoio financeiro na condução do trabalho. 


\section{LITERATURA CITADA}

CHACHALIS, D. et al. Herbicide efficacy as related to leaf morphology, epicuticular wax and spray droplets in Ipomoea spp and Jacquemontia tamnifolia. Disponível em: <http// www.nal.usda.gov>. Acesso em: 31 ago. 1999.

COLEN, D. J. Detoxification and activation of agrochemicals in plants. Pestic. Sci., v. 42, p. 209-222, 1994.

COUPLAND, D. Metabolism of glyphosate in plants. In: GROSSBARD, E.; ATKINSON, D. (Eds.). The herbicide glyphosate. London: Butterworth, 1985. p. 25-33.

COUPLAND D.; CASELEY, D. C. Presence of ${ }^{14} \mathrm{C}$ activity in root exudates and guttation fluid from Agropyron repens treated with ${ }^{14}$ C-glyphosate. New Phytol., v. 83, p. 17-22, 1979.

DELLACIOPPA, G. et al. Translocation of the precursor of 5-enolpyruvylshikimate-3-phosphate synthase into chloroplasts of higher plants in vitro. Proc. National Acad. Sci. USA, v. 83, p. 6973-6877, 1986.

DEVINE, M. D.; BANDEEN, J. D.; MCKERSIE, B. D. Temperature effects on glyphosate absorption, translocation and distribution in quackgrass (Agropyron repens). Weed Sci., v. 31, p. 461-464, 1983.

DYER, W. E. Resistance to glyphosate. In: POWLES S. B.; HOLTUM J. A. M. (Eds.). Herbicide resistance in plants. Boca Raton: CRC Press, 1994. p. 229-241.

KIRKWOOD, R. C.; MCKAY, I. Accumulation and elimination of herbicides in select crop and weed species. Pestic. Sci., v. 42, p. 241-249, 1994.

KOMOBA, D.; GENNITY, I.; SANDERMANN, H. Plant metabolism of herbicides with C-P bonds glyphosate.

Pestic. Biochem. Physiol., v. 43, p. 85-94, 1992.
MALLIK, J.; BARRY, G.; KISHORE, G. The herbicide glyphosate. Biofactors, v. 1, p. 17-25, 1989.

MCWHORTER, C. G.; JORDAN, T. N.; WILLS, G. D. Translocation of ${ }^{14} \mathrm{C}$ glyphosate in soybeans (Glycine max) and johnsongrass (Sorghum halepense). Weed Sci., v. 28, p. 113-118, 1980.

RODRIGUES, J. V. Exudation of glyphosate from wheat (Triticum aestevum) plants and its effects on interplanted corn (Zea mays) and soybean (Glycine max). Weed Sci., v. 30, p. 316-320, 1982.

SANDBERG, C. L.; MEGGITT, W. F.; PENNER, D. Absorption, translocation and metabolism of 14Cglyphosate in several weed species. Weed Res., v. 20, p. 195-200, 1980.

SATICHIVI, N. M. et al. Absorption and translocation of glyphosate isopropylamine and trimethysulfonium salts in Abutilon theophrasti and Setaria faberi. Weed Sci., v. 48, p. 675-679, 2000.

SCHULTZ, M. E.; BURNSIDE, O. C. Absorption, translocation and metabolism of 2,4-D and glyphosate in hemp dogbane (Apocynum cannabinum). Weed Sci., v. 28, p. 13-20, 1980.

SPRANKLE, P.; MEGGIT, W. F.; PENNER, D. Absorption, action and translocation of glyphosate. Weed Sci., v. 26, p. 673-676, 1975.

SPRANKLE, P. et al. Separation of glyphosate and possible metabolites by thin layer chromatography. Weed Sci., v. 26, p. 673-675, 1978.

WANAMARTA, G. D.; PENNER, D. Foliar absorption of herbicides. Weed Sci., v. 4, p. 215-231, 1989.

WILLS, G. D. Factors affecting toxicity and translocation of glyphosate in cotton. Weed Sci., v. 26, p. 509-513, 1978. 